\title{
Condition Monitoring of Cooling Tower Fans Using the Envelope Technique
}

\author{
R. Pai. ${ }^{1, a}$, Girish H. ${ }^{1, b}$ \\ ${ }^{1}$ Department of Mechanical and Manufacturing Engineering, Manipal Institute of Technology, \\ Manipal University, Manipal 576104, India \\ ahgirish789@gmail.com, ${ }^{b}$ raghuvir.pai@manipal.edu
}

\section{Keywords: Cooling tower fan, Envelope technique, Condition monitoring}

\begin{abstract}
Damaged rolling contact bearings generate vibration. These vibrations may be measured and separated from other vibrations in a machine to analyze and interpret the condition of the bearings and other associated problems. The envelope technique is used to measure the magnitude or energy distribution of the shock emitted whenever a defect in a bearing comes into contact with another surface. Frequency analysis of the enveloped signal determines the type of fault and the amplitude level, the extent of the fault. In this paper, the envelope technique is used to analyze alignment problems associated with cooling tower fans in a large refinery processing crude oil. Experimental data obtained are reported.
\end{abstract}

\section{Introduction}

Condition monitoring of machine health can be done by various methods. Signals that could be used to monitor include noise radiated by machine, temperature of bearing, oil level and nature of vibration at different locations of the machine etc. For rotating machines, vibrations picked up at proper locations are quite informative about various impending faults. Measurement and analysis of the vibration signal yields useful information from a proper analysis of the signal which is specific for the type of fault. In any maintenance problem the key is early warning based on a change in the trend. Earlier the warning better it is for the protection against possible damages. Envelope analysis has been performed in many situations in the condition monitoring of machines and equipment. It is used in the designing a condition monitoring system for detection of faults developed in many important components of machinery. Early detection of fault is very important in condition monitoring and a basic ingredient for the application of predictive maintenance (PM) and condition-based maintenance (CBM). Envelope analysis was studied and presented for several decades to detect the bearing fault. Several researchers proposed the possibility of applying Acoustic Emission (AE) in condition monitoring using envelope technique.

A proper analysis of the vibration spectrum enables us to identify the causes of vibration like unbalance, misalignment, defective anti-friction bearings, mechanical looseness, electrical causes, Aerodynamic forces etc.

A robust condition monitoring methodology for rolling element bearings that employs a novel empirical mode decomposition (EMD)-based method to eliminate high-level noise from an AE signal and a discrete wavelet packet transform (DWPT)-based envelope analysis technique to effectively search for symptoms of defective bearings is presented in Nguyena et. al. [1]. The experimental results show that the proposed method outperforms the conventional schemes by achieving up to $23.48 \%$ higher MPR values, even in a very noisy environment. Bediaga et. al. [2] review the traditional algorithms used to detect and diagnose faulty bearings in heavy-duty milling machine tool spindle heads. Different kinds of faults have been created deliberately on the bearings of a test spindle head. The prediction effectiveness of several detection methods are tested when faults are in different stages of development. In $\mathrm{Gu}$ et. al. [3], an AE sensor is used to capture the AE signals generated caused due to crack growth on a rotating shaft. The preprocessing of the AE signatures were done using the signal processing method proposed in the paper. From the FFT 
results the power spectrum was generated which in turn presented the peaks from fault frequencies Hence crack growth in rotating machinery detected by the AE sensor together with the signal processing method proposed to the results, crack growth can be considered and detected using an $\mathrm{AE}$ sensor and the signal processing method discussed. In Kim et. al. [4], the envelope analysis was enhanced with the addition of discrete wavelet transform (DWT) to reduce the noise level in AE signals. And then, the PR was calculated and compared with general envelope analysis result and the result of envelope analysis added DWT.

\section{The Envelope Technique}

The envelope technique monitors the shock pulse generated by a defective bearing. An accelerometer mounted on the bearing housing, picks up the shock pulse and converts it into an electrical signal. The magnitude of the pulse depends on the extent of the defect and repetition frequency will depend on the type of defect.

Defects associated with a rolling element bearing are associated with a unique frequency depending on bearing geometry and machine speed. However, it is not possible to identify bearing frequencies in a normal vibration spectrum because bearing defect frequencies are similar to frequencies associated with defects in the rotating assembly of a machine. But, the shock pulse due to impact between a defect and another surface appears as a short burst of energy over a wide frequency range. This excites natural resonant frequencies of the bearing. The pulses appear as a series of decaying wave forms, whose frequencies correspond to bearing resonance frequencies but the repetition rate corresponds to appropriate bearing defect frequency. The bursts of narrow band bearing signal are separated from the low frequency rotor vibration and the high frequency random noise using a band-pass filter. The signal is then rectified and demodulated to give the signal at one of the defect frequencies. The type of fault and the amplitude level of the extent of fault can then be determined from a frequency analysis of the signal.

Envelope spectrum helps valid measurements to be made in high noise and vibration environments. Since it looks at relative height of the defect frequency above carpet level and because both are subjected to the same attenuation across joints, the ratio is independent of transducer location. Enveloping is also independent of load on the machine. A change in load will change the defect frequency and carpet level in the same ratio. A definite pattern in the envelope spectra is observed from a bearing in good condition and that which is on the verge of failure.

\section{Condition Monitoring}

The vibration data from the cooling tower fan motors are taken on a monthly basis because they are non-critical machines and there being seven such units in the cooling tower. Horizontal, Vertical and axial vibration measurements on both the drive (DE) and non-drive end (NDE) bearings are taken using the SKF MICROLOG CMV A55 [5] condition monitoring unit. The data collected is stored in the unit and then taken to the condition monitoring cell where it is analyzed using the SKF PRISM 4 [6] PC based software for condition monitoring.

Fig. 1 shows a trend chart. The trend line for the Motor Drive End (MDE) Horizontal (H) velocity shows a gradual increase in vibration level from 5 October 1998. To ascertain whether the vibration level is exceeding the alert high level, another observation is made in mid-November 98. Actually there is a small drop in vibration level. This may be attributed to a change in position of fixed machine parts due to thermal expansion. Between mid-November and mid-December 98, the vibration level is again on the increase. It overshoots the alert high level after 1 January 1999. The data points are not recorded here in the trend chart, although measurements were made. On 1st February 1999 the vibration level reaches about $8.771 \mathrm{~mm} / \mathrm{sec}$ which is near about the danger high level of $10 \mathrm{~mm} / \mathrm{sec}$. On analyzing the spectrum it was observed that the axial vibration levels are about half as much as the radial vibration levels as seen from the last measurements quick report (Table 1.) The amplitudes also peak at the $1 \mathrm{x}$ frequency, when the measurements were made on two 
separate dates (Fig. 3 and Fig. 4) indicating misalignment commonly associated with faulty initial alignment. Misalignment causes high stresses that damage the bearings and couplings. To check whether the vibration data recorded was due to misalignment, the motor was decoupled from the propeller shaft and run on load. The vibration levels were within the prescribed limits for the motor and therefore it was concluded that the motor was all right. The alignment was done and the vibration measurements were made. The spectrum (Fig. 2) showed that the vibration level had come down to $4.563 \mathrm{~mm} / \mathrm{sec}$ in the horizontal direction and $3.091 \mathrm{~mm} / \mathrm{sec}$ in the axial direction (Table 1.)

\section{CT -I: CT FAN:GB8241B:MDE H VEL}

08-Feb-99 12:45:40
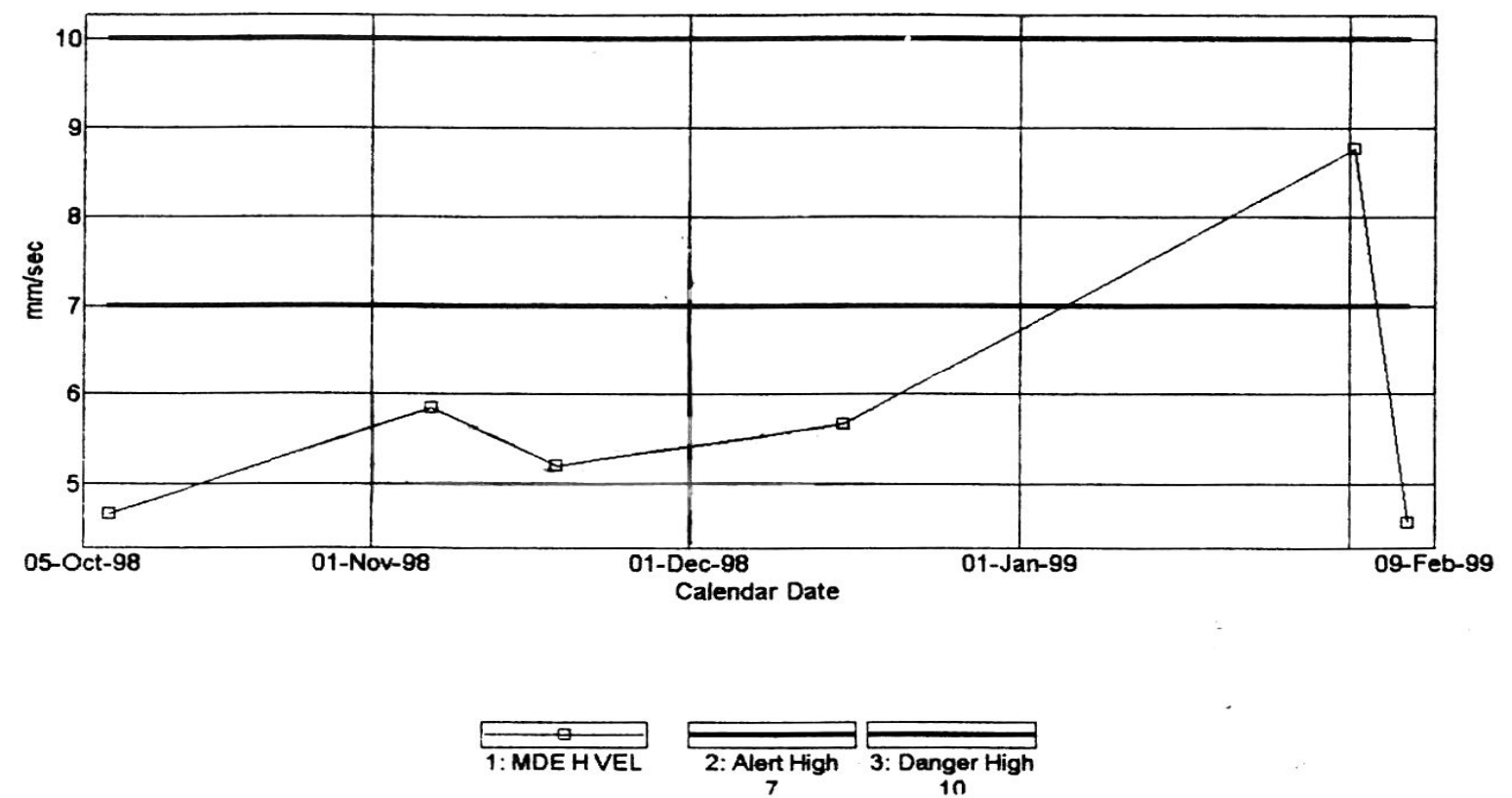

Fig. 1 Trend chart.

CT -I: CT FAN:GB8241B:MDE H VEL

08-Feb-99 12:48:54

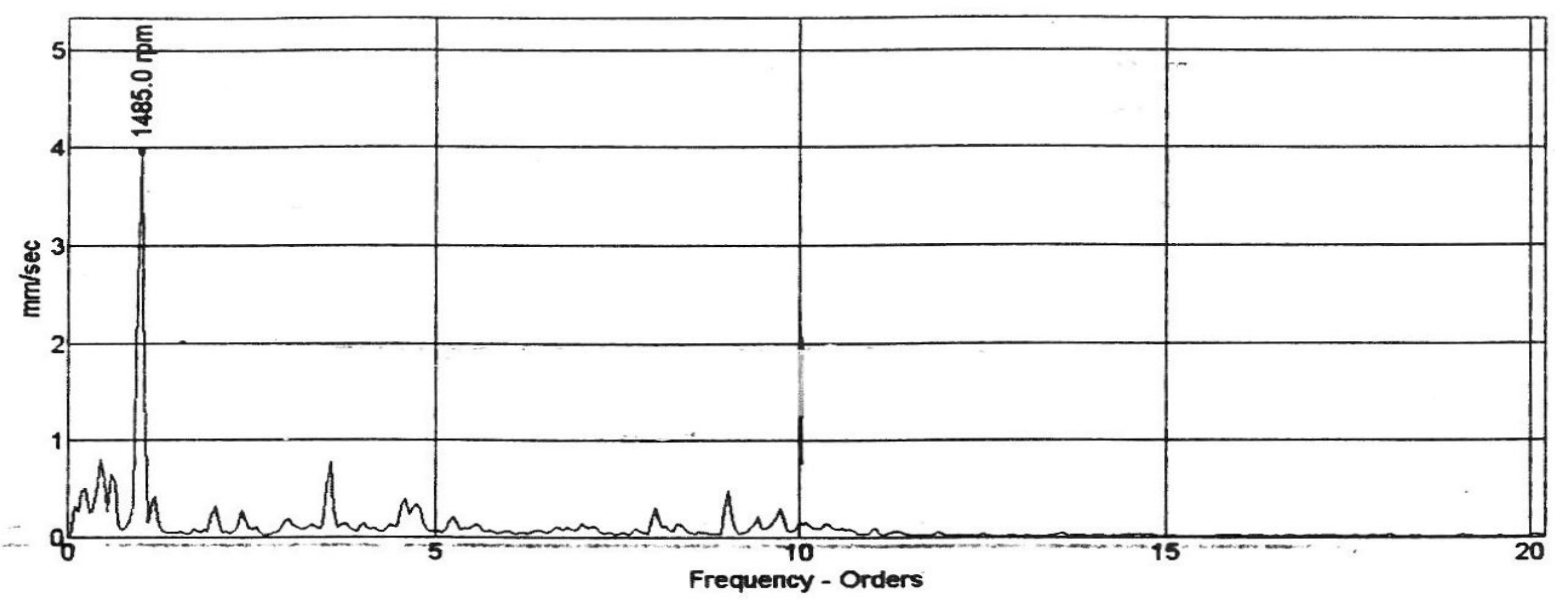

1: MDE HVEL

city (Ace to Vel) (RMS)

Q6-Feb-99 14:40:07

Fig. 2 Vibration spectrum. 
CT -I: CT FAN:GB8241B:MDE H VEL

08-Feb-99 12:54:55
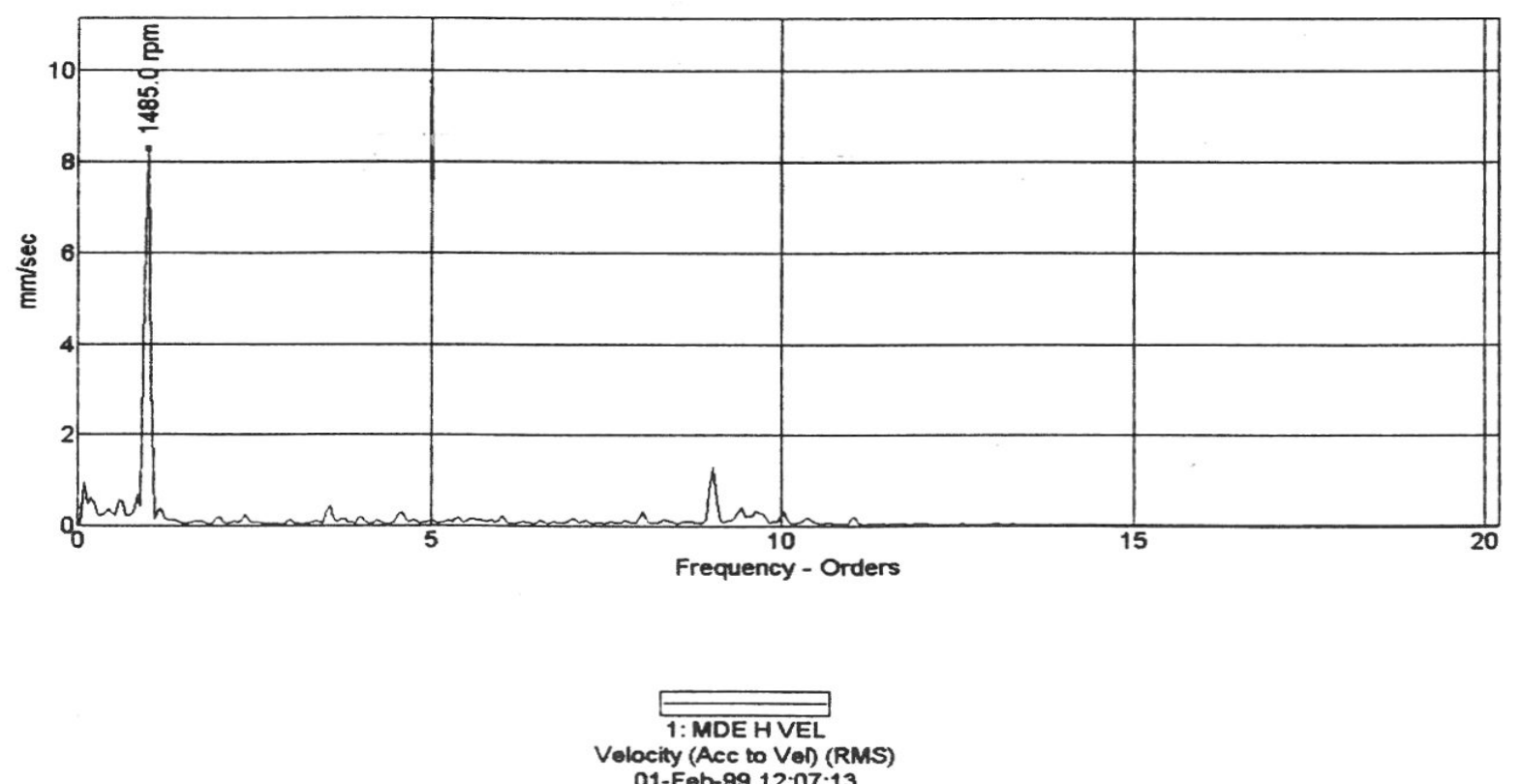

Fig. 3 Vibration spectrum.

CT -I: CT FAN:GB8241B:MDE H ENV

06-Feb-99 16:58:30
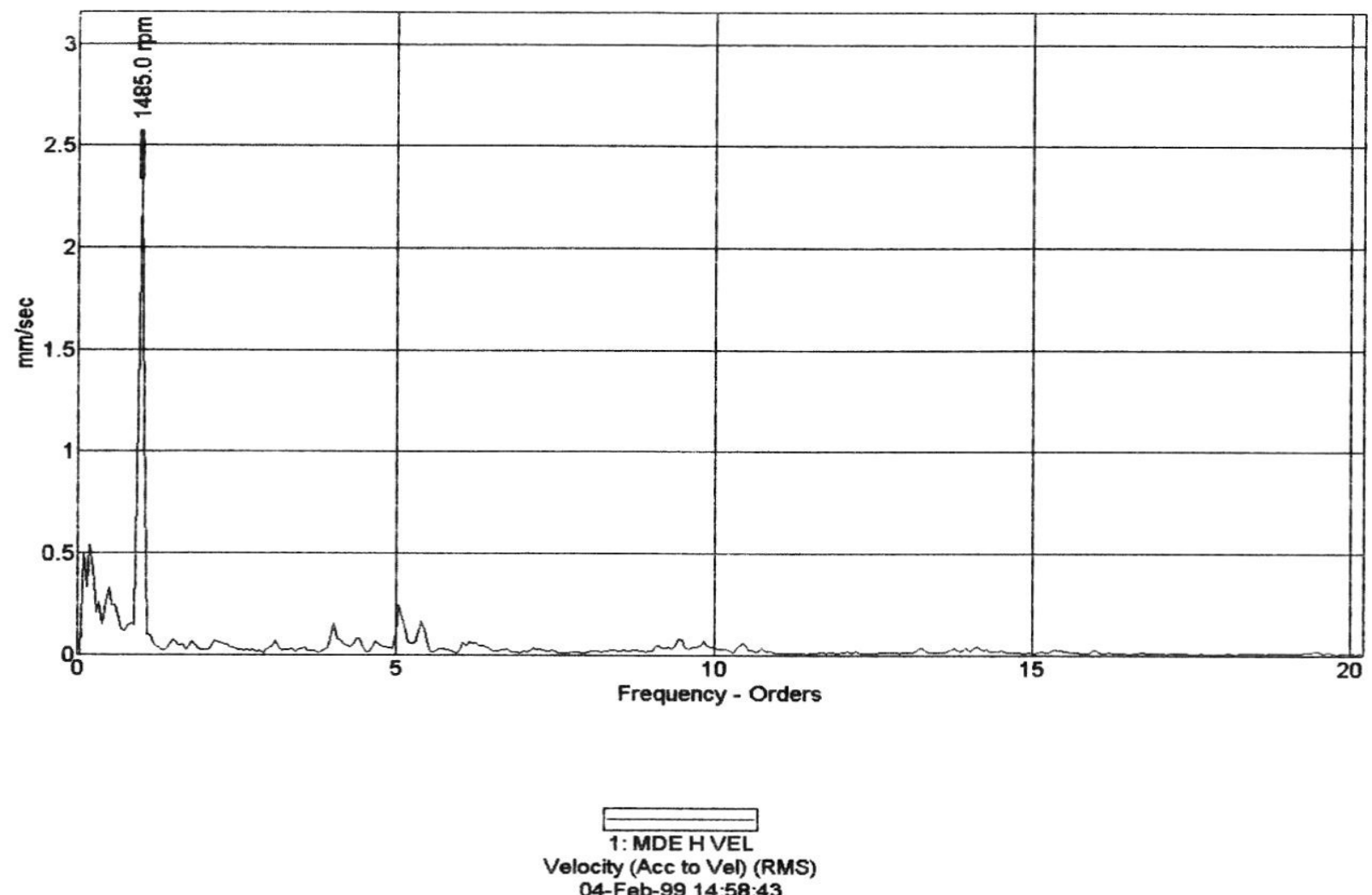

Fig. 4 Vibration spectrum. 
Table 1. Last measurement quick report.

\begin{tabular}{|l|c|c|c|c|c|}
\hline ID & Units & Date & Last Value & $\begin{array}{c}\text { Previous } \\
\text { Value }\end{array}$ & Percent Change \\
\hline GB8241B (Machine Code) & $\mathrm{mm} / \mathrm{sec}$ & 06-Feb-99 & 4.563 & 8.771 & -48.0 \\
\hline MDE H VEL & Gs Env & 06-Feb-99 & 2.297 & 8.045 & -71.5 \\
\hline MDE H ENV & $\mathrm{mm} / \mathrm{sec}$ & 06-Feb-99 & 3.478 & 4.682 & -25.7 \\
\hline MDE V VEL & $\mathrm{mm} / \mathrm{sec}$ & 06-Feb-99 & 3.091 & 5.387 & -42.6 \\
\hline
\end{tabular}

\section{Conclusion}

- Removal of electric supply did not immediately diminish the vibration levels, cause of vibration is essentially of mechanical origin.

- Initial alignment and balance should be accurate to avoid future operation problems.

- Misalignment can cause bearing distress leading to early failure.

- The envelope technique helps measurements to be made in high noise and vibration environments.

\section{References}

[1] P. Nguyena, M. S. Kang, J. M. Kim, B. H. Ahn, J. M. Ha, B. K. Choi, Robust condition monitoring of rolling element bearings using de-noising and envelope analysis with signal decomposition techniques, Expert Syst. Appl. 42(22) (2015) 9024-9032.

[2] I. Bediaga, X. Mendizabal, A. Arnaiz, J. Munoa. Ball bearing damage detection using traditional signal processing algorithms, IEEE Instru. Meas. Mag. 16(2) (2013) 20-25.

[3] D. Gu, J. Kim, T. Kelimu, S. C. Huh, B. K. Choi, Evaluation of the use of envelope analysis and DWT on AE signals generated from degrading shafts, Mater. Sci. Eng. B. 177(19) (2012) 1683-1690.

[4] B. S. Kim, D. S. Gu, J. G. Kim, Y. C. Kim, B. K. Choi, Rolling element bearing fault detection using acoustic emission signal analyzed by envelope analysis with discrete wavelet transform, Eng. Asset Lifecycle Manage. (2010) 596-602.

[5] SKF MICROLOG CMV A55 Catalogue, www.skf.com

[6] SKF PRISM 4 Software Catalogue, www.skf.com 